\title{
GERAÇÃO E COMPOSIÇÃO GRAVIMÉTRICA DE RESÍDUOS SÓLIDOS URBANOS EM MUNICÍPIOS DO SEMIÁRIDO DE ALAGOAS
}

\section{Generation and Gravimetric Composition of Urban Solid Waste in Municipalities of the Semi-Arid Region of Alagoas}

José Rayan Eraldo Souza Araújo ${ }^{1}$, João Henrique Barbosa da Silva ${ }^{1}$, Mateus Costa Batista ${ }^{2}$, Khyson Gomes Abreu ${ }^{1}$, Erico Dos Anjos Dantas ${ }^{1}$, Lucas Firmino da Silva Medeiros ${ }^{1}$, Diego Alves Monteiro da Silva ${ }^{1}$, João Vitor Andrade Magalhães $^{1}$, João Paulo de Oliveira Santos ${ }^{1}$

${ }^{1}$ Centro de Ciências Agrárias, UFPB - Universidade Federal da Paraíba, Areia - Paraíba. ${ }^{2}$ Centro de Tecnologia e Recursos Naturais, UFCG - Universidade Federal de Campina Grande, Campina Grande - Paraíba.

E-mail: rayanccaufpb@gmail.com, henrique485560@gmail.com, khysonabreu@gmail.com, erico anjos@ @hotmail.com, lucasfsmedeiros@hotmail.com, diegoalves1903@gmail.com, v.andrademagalhaes@gmail.com, jpos@agro.adm.br, matheus1384@hotmail.com

RESUMO - A geração de resíduos sólidos urbanos (RSU) tem crescido de forma expressiva nas últimas décadas, principalmente devido ao aumento populacional e das áreas urbanas. Dessa forma, torna-se necessário conhecer a quantidade e a composição desses materiais, principalmente em pequenos municípios, onde a gestão desses resíduos ainda é deficitária. Nesse sentido, o presente estudo objetiva analisar a produção de resíduos sólidos urbanos e a sua gravimetria em oito municípios do sertão de Alagoas. Como fonte de dados, utilizou-se o Plano Estadual de Resíduos Sólidos do Estado de Alagoas - Região Sertão e informações econômicas do Instituto Brasileiro de Geografia e Estatística. Uma Análise de Componentes Principais (ACP) foi empregada. Os municípios se caracterizaram por apresentar baixa população urbana e baixos valores de PIB per capita. Foi observada elevada variabilidade para geração diária de RSU, com a predominância da fração orgânica, seguida pelo plástico. Em contrapartida, observou-se baixos percentuais de metais (<5\%). A ACP explicou $69.2 \%$ da variância original dos dados nos dois primeiros eixos e evidenciou a forte associação entre aspectos econômicos e a composição gravimétrica dos resíduos. Visto a grande quantidade de material orgânico presente nos resíduos da área de estudo, ações de aproveitamento e destinação adequada dessa fração, como a compostagem, devem ser incentivadas.

Palavras-chave: aproveitamento de resíduos, degradação ambiental, destinação.

ABSTRACT - The generation of solid urban waste (MSW) has grown significantly in recent decades, mainly due to the increase in population and urban areas. Thus, it is necessary to know the quantity and composition of these materials, especially in small municipalities, where the management of this waste is still deficient. In this sense, the present study aims to analyze the production of solid urban waste and its gravimetry in eight municipalities in the sertão of Alagoas. As a source of data, the State Solid Waste Plan of the 
State of Alagoas - Sertão Region and economic information from the Brazilian Institute of Geography and Statistics were used. A Principal Component Analysis (PCA) was employed. The municipalities were characterized by a low urban population and low GDP per capita values. High variability was observed for the daily generation of MSW, with the predominance of the organic fraction, followed by plastic. On the other hand, low percentages of metals $(<5 \%)$ were observed. The ACP explained $69.2 \%$ of the original variance of the data in the first two axes and evidenced the strong association between economic aspects and the gravimetric composition of the residues. In view of the large amount of organic material present in the residues of the study area, actions for the use and proper disposal of this fraction, such as composting, should be encouraged.

Keywords: utilization of residues, environmental degradation, destination.

\section{INTRODUÇÃO}

O planeta Terra tem apresentado um expressivo aumento populacional e das áreas urbanizadas, resultando na geração de grandes quantidades de resíduos sólidos (LISSAH et al., 2021). Esse aumento é uma questão problemática e preocupante em todo o mundo, especialmente nos centros urbanos (ABDELSHAFY; MANSOUR, 2018), com destaque para os países em desenvolvimento, que apresentam pouca estrutura para o manejo desses materiais (LISSAH et al., 2021).

Os resíduos sólidos urbanos (RSU) são os resíduos resultantes da atividade doméstica e comercial dos ambientes urbanos; estes apresentam variações substanciais em sua composição e classificação dependendo do município de que são oriundos, embora de modo geral são constituídos por frações biodegradáveis e não biodegradáveis de materiais orgânicos e inorgânicos, respectivamente (NANDA; BERRUTI, 2020).

Previsões indicam que até 2025, a produção global de resíduos sólidos em áreas urbanas aumentará de 3.500 .000 toneladas para 6.100.000 toneladas por dia (MAKARICHI et al., 2018). Se mal gerenciados, os RSUs podem causar degradação da qualidade do ar, do solo e da água, tornar-se um problema de saúde pública, contribuir para as mudanças climáticas e para a deterioração da fauna e flora aquática e terrestre (RAMACHANDRA et al., 2018; JOTAWORN et al., 2021).

Dotado de uma população estimada de 209,3 milhões de habitantes, o Brasil gerou cerca de 78,4 milhões de toneladas de resíduos sólidos urbanos em 2017 (COSTA; DIAS, 2020). No país, os pequenos municípios são os que enfrentam os maiores desafios par a gestão desses resíduos, destaca-se que a maioria dos municípios brasileiros é de pequeno porte (possui até 100 mil habitantes) e representam a maior parcela da população total e a maior parcela dos resíduos produzidos (DEUS et al., 2020). Como um país de renda média, o Brasil apresenta diferenças de grande parte dos países em desenvolvimento no que diz respeito a geração per capita de resíduos. Todavia, em grande parte de seu território, a qualidade da gestão dos resíduos é tão precária quanto a alguns outros países de baixa renda (COSTA; DIAS, 2020).

Conhecer a quantidade e a composição dos resíduos sólidos urbanos é uma ação fundamental para a determinação do manejo e gerenciamento adequados desses materiais (ABDEL-SHAFY; MANSOUR, 2018). Ademais, compreender a natureza dos resíduos sólidos urbanos em um município é fundamental para a sua gestão tecnológica (CUDJOE; ACQUAH, 2021). Os resíduos sólidos urbanos são extremamente diversificado em sua composição, seus principais componentes globalmente são papel, material orgânico, metal, vidro, plástico, madeira, têxtil, e outros (ABDEL-SHAFY; MANSOUR, 2018; (MAKARICHI et al., 2018; CUDJOE; ACQUAH, 2021). Todavia, essas frações são variáveis e devem ser conhecidas para uma destinação correta desses resíduos, além de refletirem o grau de desenvolvimento e consumo local (ABDEL-SHAFY; MANSOUR, 2018; AZEVEDO et al.,, 2020). Ação ainda mais importante em regiões carentes de estudos com esse enfoque, como os pequenos municípios do Semiárido do Brasil (CARTAXO et al., 2019; SANTOS et al., 2020). 
Nesse sentido, o presente estudo objetiva analisar a produção de resíduos sólidos urbanos e a gravimetria desses materiais em oito municípios do sertão de Alagoas. Buscando-se assim, subsidiar ações que conduzam ao aproveitamento e a destinação correta desses materiais localmente.

\section{MATERIAL E MÉTODOS}

A pesquisa foi conduzida em oito municípios do sertão alagoano: Água Branca, Canapi, Delmiro Gouveia, Inhapi, Mata Grande,

Figura 1. Localização dos municípios em estudo.

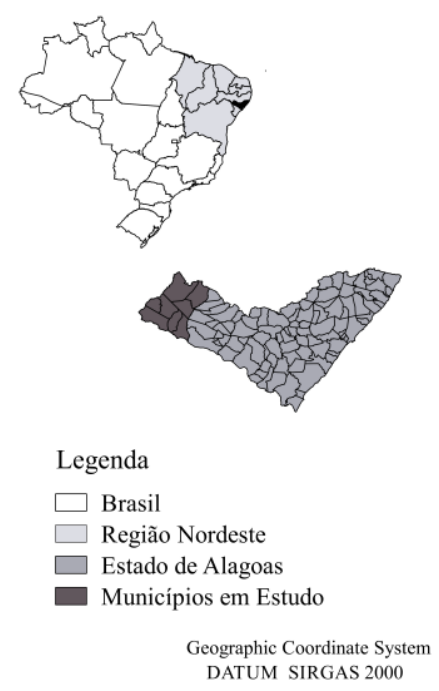

Após a obtenção, os dados foram organizados em tabelas, utilizando-se o software Microsoft Excel ${ }^{\circledR}$. A matriz de dados também foi submetida a uma análise multivariada através de Análise de Componentes Principais (ACP), buscando-se assim, reduzir o número de variáveis, mantendo, no entanto, o mesmo nível de informação oferecido pelo conjunto total das variáveis originais. ACP é uma técnica de redução de dados estatísticos multivariados em que componentes principais são calculadas por combinações lineares das variáveis iniciais; uma das vantagens desse método, além da redução dimensional, é que esses componentes principais são ortogonais entre si, portanto, não carregam informações redundantes (BAYARD et al., 2018). Para a confecção da Análise de Componentes
Olho d'Água do Casado, Pariconha e Piranhas (Figura 1). Todos os municípios estão inseridos nos domínios do Semiárido brasileiro e do bioma Caatinga (IBGE, 2018).

Os dados relativos à geração de resíduos sólidos urbanos e sua gravimetria na área de estudo foram obtidos através do Plano Estadual de Resíduos Sólidos do Estado de Alagoas - Região Sertão (PIGIRS, 2016). Dados econômicos foram obtidos juntos ao Instituto Brasileiro de Geografia e Estatística (IBGE, 2018).

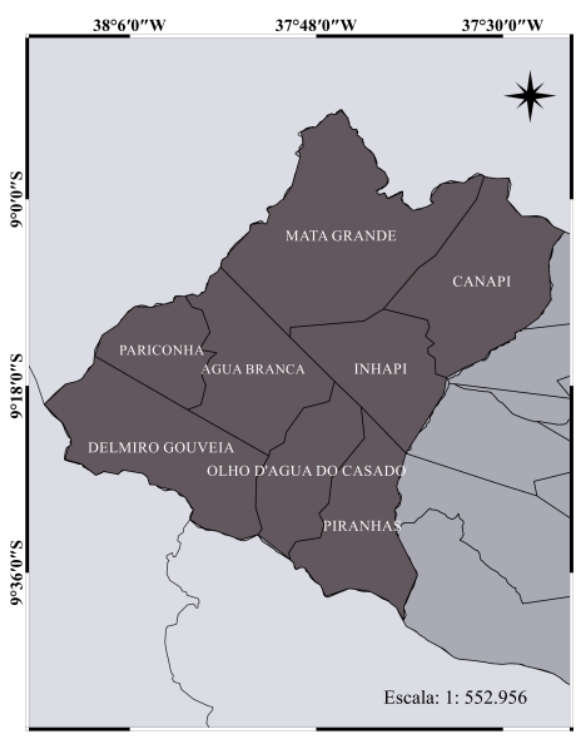

Principais foi utilizado o software $R \quad(R$ DEVELOPMENT CORE TEAM, 2019).

\section{RESULTADOS E DISCUSSÃO}

Os municípios em estudo, com exceção de Delmiro Gouveia e Piranhas, apresentam população urbana inferior a $10 \mathrm{mil}$ habitantes (Tabela 1). Situação comum no Semiárido brasileiro, em que ainda há uma grande concentração populacional nas áreas rurais (PETERSEN; SILVEIRA, 2017). Baixos valores de PIB per capita também foram observados, com valores inferiores ao PIB per capita estadual (R\$ 16.376) e nacional ( $R \$ 33.593,82)$ para o mesmo período (IBGE, 2018). Resultados que evidenciam a vulnerabilidade socioeconômica presente, concomitante com poucas opções locais de geração de renda (PETERSEN; SILVEIRA, 2017). 
Tabela 1. População urbana, PIB per capita e estimativa de geração diária de RSU nos municípios em estudo.

\begin{tabular}{lrrr}
\hline \multicolumn{1}{c}{ Município } & $\begin{array}{c}\text { População Urbana Projetada } \\
\text { (2016) }\end{array}$ & PIB per capita & $\begin{array}{c}\text { Geração de resíduos estimada } \\
\text { (t/dia) }\end{array}$ \\
\hline Água Branca & 5.502 & $7.004,81$ & 3,14 \\
Canapi & 6.310 & $6.629,41$ & 3,6 \\
Delmiro Gouveia & 35.909 & $10.039,86$ & 23,34 \\
Inhapi & 7.631 & $6.413,19$ & 4,35 \\
Mata Grande & 6.040 & $8.863,29$ & 3,44 \\
Olho d'Água do Casado & 4.118 & $7.602,31$ & 2,35 \\
Pariconha & 3.091 & $6.971,88$ & 1,76 \\
Piranhas & 14.853 & $9.888,19$ & 8,47 \\
\hline
\end{tabular}

Fonte: Adaptado do Plano Intermunicipal de Gestão Integrada de Resíduos Sólidos da Região Sertão, Alagoas (2016) e do Instituto Brasileiro de Geografia e Estatística (IBGE, 2018).

Elevada variabilidade foi observada para geração diária de RSU, com valores oscilando de 1,76 (Pariconha) a 23,34 toneladas/dia (Delmiro Gouveia). Essa diferença acentuada é derivada da maior ou menor população urbana e seus hábitos de consumo e descarte (ALFAIA et al., 2017; CARTAXO et al., 2019). A matéria orgânica foi o principal constituinte dos resíduos sólidos urbanos nos municípios em estudo (Tabela 2), tendência comum para esses materiais em países em desenvolvimento como o Brasil (MAKARICHI et al., 2018). Já em países industrializados, a fração inerte constitui aproximadamente 70\% dos resíduos (AZEVEDO et al., 2020).

Destaca-se ainda, que em quase a totalidade dos municípios essa fração foi superior a $50 \%$, chegando a compor mais de $3 / 4$ dos RSUs em Olho d'Água do Casado. Comportamento semelhante já foi relatado para outros municípios do semiárido alagoano, como Poço das
Trincheiras (CARTAXO et al., 2019) e Dois Riachos (SANTOS et al., 2020).

Diante dessa conjuntura, ações de uso dos resíduos orgânicos devem ser estudadas e incentivadas; entre essas ações destacam-se a compostagem, digestão anaeróbica, produção de ração animal e outras operações de reprocessamento de materiais orgânicos; dentre estas, a compostagem, quando realizada de maneira adequada, é uma excelente opção de gestão de resíduos orgânicos, gerando diversos benefícios, entre eles a redução da produção de gases de efeito estufa e a melhoria da qualidade do solo quando usada como corretivo agrícola (WEl et al., 2017).

O plástico se mostrou como um importante constituinte dos RSUs, chegando a contribuir com $25,37 \%$ do quantitativo total em Mata Grande.

Tabela 2. Composição Gravimétrica (\%) dos resíduos sólidos urbanos dos municípios em estudo.

\begin{tabular}{lrrrrrr}
\hline \multicolumn{1}{c}{ Município } & Matéria Orgânica & Papel/Papelão & \multicolumn{1}{c}{ Metal } & Plástico & Vidro & Outros \\
\hline Água Branca & 60,63 & 10,02 & 3,16 & 13,36 & 1,93 & 10,9 \\
Canapi & 50,85 & 18,2 & 0,51 & 10,03 & 1,53 & 18,88 \\
Delmiro Gouveia & 58,49 & 6,4 & 2,14 & 15,03 & 5,27 & 12,67 \\
Inhapi & 57,9 & 17,4 & 1,5 & 12,6 & 3,9 & 6,3 \\
Mata Grande & 38,81 & 8,96 & 7,46 & 25,37 & 8,96 & 10,45 \\
Olho d'Água do Casado & 75,44 & 3,33 & 3,33 & 6,49 & 2,63 & 8,77 \\
Pariconha & 59,72 & 7,64 & 3,47 & 9,72 & 2,78 & 16,67 \\
Piranhas & 57,69 & 7,25 & 2,5 & 13,75 & 5 & 13,81 \\
\hline \multicolumn{1}{c}{ Média } & 57,4 & 9,9 & 3,01 & 13,3 & 4 & 12,3 \\
\hline
\end{tabular}

Fonte: Adaptado do Plano Intermunicipal de Gestão Integrada de Resíduos Sólidos da Região Sertão, Alagoas (2016). 
A categoria outros, que abriga materiais como pedaços de trapos, tecidos, madeiras, borrachas, couros e qualquer outro resíduo que não se enquadre nos cinco outros componentes definidos, também apresentou uma contribuição significativa, com uma média geral de 13,3\% (Tabela 2). Ademais, mesmo dotada de ganhos ambientais e econômicos, a prática da reciclagem ainda não foi totalmente disseminada no Brasil, onde menos de um terço das cidades possuem programas ativos de reciclagem (CONKE, 2018). Um outro gargalo na gestão de RSUs é a coleta e a destinação final desses materiais, enquanto no Brasil a cobertura de coleta é da ordem de $91,24 \%$, na região Nordeste esse número é de $79,06 \%$, com o agravante que $74,97 \%$ dos municípios nordestinos ainda utilizam sistemas de disposição final inadequada, como os lixões (COSTA; DIAS; 2020). Além disso, o despejo de lixo em local não autorizado também é uma das práticas comuns em cidades urbanizadas de países em desenvolvimento; a deposição

Figura 2. Análise de Componentes Principais (ACP). inadequada de todas as categorias de resíduos, incluindo os RSUs, leva a problemas ambientais, como a contaminação da água e do solo devido ao processo de degradação e lixiviação dos constituintes desses materiais (RAMACHANDRA et al., 2018).

Nesse sentido, para extinguir a prática de despejo irregular de resíduos, o Brasil desenvolveu uma legislação para o tratamento e destinação de resíduos sólidos baseado no protocolo europeu, a Política Nacional de Resíduos Sólidos (PNRS), instituída pela Lei no $12.305 / 2010$, e que previa o fechamento de 2.906 lixões até agosto de 2014 , no entanto essa meta não foi alcançada e o prazo foi prorrogado (LINO; ISMAIL, 2018). Na área de estudo desse trabalho, os municípios se uniram e criaram o Consórcio Regional de Resíduos Sólidos do Sertão de Alagoas (CRERSSAL), no qual uma das finalidades foi inativar os lixões locais e destinar os resíduos para um aterro sanitário.

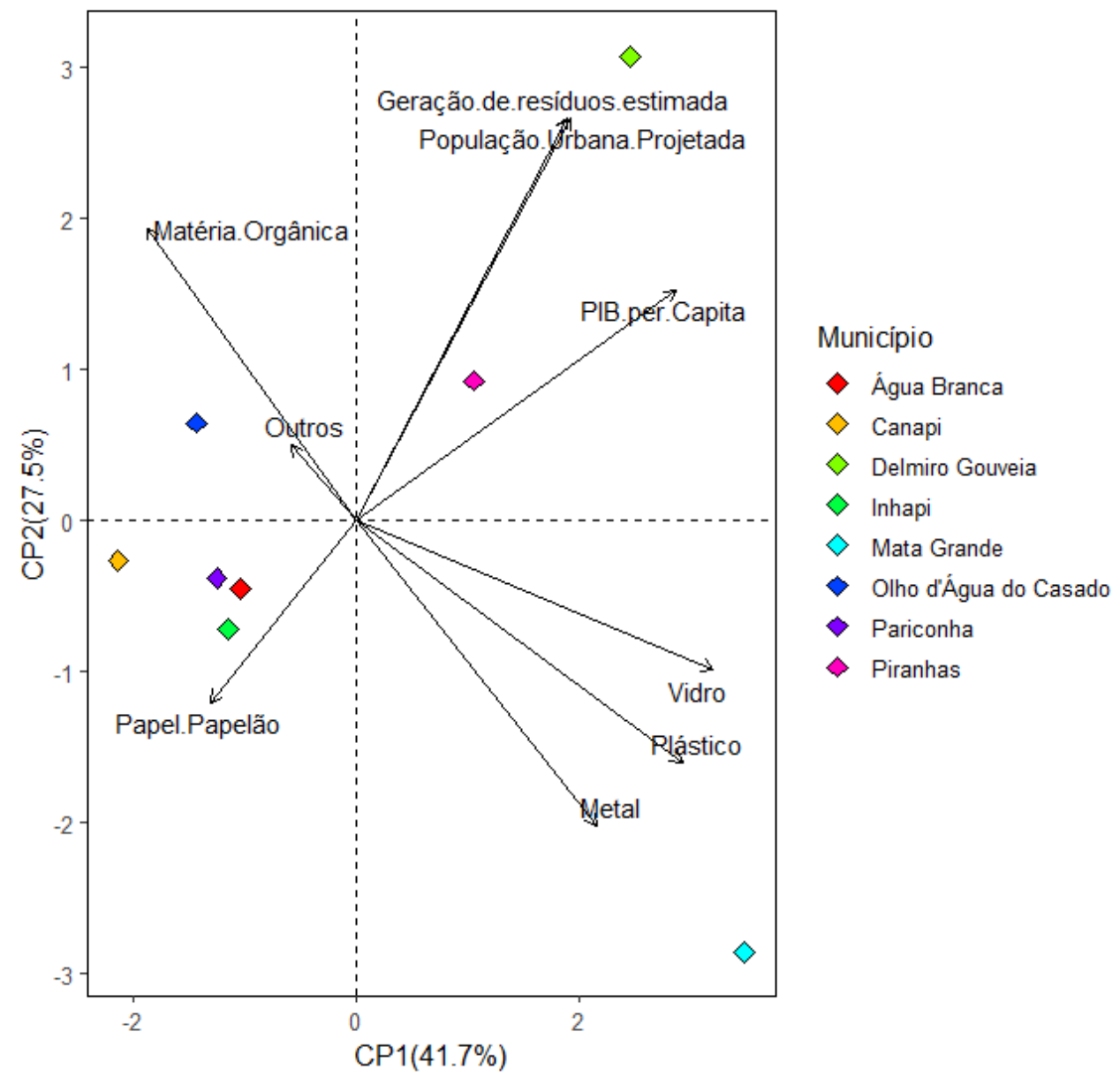


A análise de componentes principais (ACP) (Figura 2) explicou 69.2\% da variância original dos dados nos dois primeiros eixos (CP1 e CP2). No eixo 1, responsável por aglutinar $41.7 \%$ da explicação dos dados, observou-se associação positiva entre vidro $(r=0,92)$, plástico $(r=0,84)$, metal $(r=0,62)$ e PIB per capita $(r=0,83)$ em contraste com a quantidade de matéria orgânica $(r=-0,54)$. A composição dos RSUs varia significativamente de um município para outro e de país para país, variação essa que depende principalmente do estilo de vida, da situação econômica, dos regulamentos de gestão de resíduos e da estrutura industrial (ABDEL-SHAFY; MANSOUR, 2018). Ao mesmo tempo, o volume de resíduos gerados e sua composição estão intimamente ligados ao PIB (MAKARICHI et al., 2018).

Nesse sentido, a ACP permitiu a formação de dois agrupamentos. Um formado pelos municípios de Delmiro Gouveia, Piranhas e Mata Grande, com maior percentual de vidro, plástico e metal na composição de seus RSUs; e outro cluster formado por Água Branca, Canapi, Inhapi, Olho d'Água do Casado e Pariconha, que apresentaram maior percentual de matéria orgânica, papel/papelão e outros.

Os resultados aqui observados, explicitam que municípios com maior PIB per capita, como por exemplo, Delmiro Gouveia, Piranhas e Mata Grande, apresentam maior tendência a apresentarem um menor percentual de matéria orgânica em seus RSUs, em contrapartida, a porcentagem de materiais como vidro, plástico e metal são aumentadas. Comportamento esse também observado em outras partes do mundo, principalmente em países desenvolvidos (HETTIARACHCHI et al., 2018)

O eixo 2 , que reúne $27.5 \%$ da explicação da variância original, apresentou a associação positiva entre a geração diária de resíduos estimada $(r=0,77)$ e a população urbana projetada $(r=0,77)$. Comportamento esperado, visto que ao passo que se tem uma maior população, espera-se também uma maior geração de resíduos. Todavia, essa geração de resíduos pode sofrer influência de outros fatores, principalmente os econômicos, de modo que, duas cidades com a mesma população podem apresentar geração diárias de resíduos bem diferentes (ABDEL-SHAFY; MANSOUR, 2018; CARTAXO et al., 2019).

\section{CONSIDERAÇÕES FINAIS}

A área em estudo apresenta forte variabilidade na geração diária de resíduos sólidos urbanos.

A matéria orgânica é a principal fração presentes nos resíduos, seguida pelo plástico.

A análise de componentes principais (ACP) evidenciou a forte associação entre aspectos econômicos e a composição gravimétrica dos resíduos nos municípios em estudo.

Ações de aproveitamento e destinação adequada de resíduos orgânicos, como a compostagem, devem ser incentivadas.

\section{REFERÊNCIAS}

ABDEL-SHAFY, H. I.; MANSOUR, M. S. M. Solid waste issue: Sources, composition, disposal, recycling, and valorization. Egyptian Journal of Petroleum, v. 27, n. 4, p. 1275-1290, 2018. DOI: https://doi.org/10.1016/i.ejpe.2018.07.003

ALFAIA, R. G. S. M.; COSTA, A. M.; CAMPOS, J. C. Municipal solid waste in Brazil: A review. Waste Management \& Research, v. 35, n. 12, p. $1195-$ 1209, 2017. DOI: https://doiorg/10.1177\%2F0734242X17735375

AZEVEDO, A. R.; COUTINHO, R. A. D. S.; PEREIRA, C. R.; CECCHIN, D. Characterization of solid waste of restaurant and its energy generation potential: case study of Niterói, RJ, Brazil. Biomass Conversion and Biorefinery, v. 2020, p. 1-10, 2020. DOI: https://doi-org/10.1007/s13399-02000705-1

BAYARD, R.; BENBELKACEM, H.; GOURDON, R.; BUFFIÈRE, P. Characterization of selected municipal solid waste components to estimate their biodegradability. Journal of Environmental Management, v. 216, p. 4-12, 2018. DOI: https://doi-

org.ez15.periodicos.capes.gov.br/10.1016/i.jenv man.2017.04.087

CARTAXO, P. H. A.; LUNA, I. R. G.; NASCIMENTO, I. R. S.; SILVA, M. R.; SILVA, K. A.; SANTOS, J. P. O. Geração de resíduos sólidos urbanos no semiárido brasileiro: análise de sustentabilidade a partir da aplicação da pegada ecológica. TecnoLógica, v. 23, n. 2, p. 87-92, 2019. DOI: http://dx.doi.org/10.17058/tecnolog.v23i2.13077 
CONKE, L. S. Barriers to waste recycling development: Evidence from Brazil. Resources, Conservation and Recycling, v. 134, p. 129-135, $2018 . \quad$ DOI: https://doiorg.ez15.periodicos.capes.gov.br/10.1016/i.resco nrec.2018.03.007

COSTA, I. M.; DIAS, M. F. Evolution on the solid urban waste management in Brazil: A portrait of the Northeast Region. Energy Reports, v. 6, p. 878-884, 2020.2 DOI: https://doi.org/10.1016/j.egyr.2019.11.033

CUDJOE, D.; ACQUAH, P. M. Environmental impact analysis of municipal solid waste incineration in African countries. Chemosphere, v. 265, p. e129186, 2021. DOI: https://doi.org/10.1016/j.chemosphere.2020.129 $\underline{186}$

DEUS, R. M.; MELE, F. D.; BEZERRA, B. S.; BATTISTELLE, R. A. G. A municipal solid waste indicator for environmental impact: Assessment and identification of best management practices. Journal of Cleaner Production, v. 242, p. e118433, 2020. DOI: https://doiorg.ez15.periodicos.capes.gov.br/10.1016/i.jclepr $\underline{0.2019 .118433}$

HETTIARACHCHI, H.; MEEGODA, J. N.; RYU, S. Organic waste buyback as a viable method to enhance sustainable municipal solid waste management in developing countries. International Journal of Environmental Research and Public Health, v. 15 , n. 11 , p. 2483, 2018. DOI: https://doi.org/10.3390/ijerph15112483

IBGE - Instituto Brasileiro de Geografia e Estatística. @Cidades. (2018). Disponível em: https://cidades.ibge.gov.br/brasil/. Acesso em 10 de maio de 2021.

JOTAWORN, $\quad$ S.; NITIVATTANANON, V.; KUSAKABE, K.; XUE, W. Partnership towards Synergistic Municipal Solid Waste Management Services in a Coastal Tourism SubRegion. Sustainability, v. 13, n. 1, p. e397, 2021. DOI: https://doi.org/10.3390/su13010397

LINO, F. A. M.; ISMAIL, K. A. R. Evaluation of the treatment of municipal solid waste as renewable energy resource in Campinas, Brazil. Sustainable Energy Technologies and Assessments, v. 29, p.
19-25, 2018. DOI: https://doiorg.ez15.periodicos.capes.gov.br/10.1016/i.seta. $\underline{2018.06 .011}$

LISSAH, S. Y.; AYANORE, M. A.; KRUGU, J. K.; ABERESE-AKO, M.; RUITER, R. A. Managing urban solid waste in Ghana: Perspectives and experiences of municipal waste company managers and supervisors in an urban municipality. PloS one, v. 16, n. 3, p. e0248392, 2021.

DOI:

https://doi.org/10.1371/journal.pone.0248392

MAKARICHI, L.; JUTIDAMRONGPHAN, W.; TECHATO, K. The evolution of waste-to-energy incineration: A review. Renewable and Sustainable Energy Reviews, v. 91, p. 812-821, 2018. DOI:

https://doi.org/10.1016/i.rser.2018.04.088

NANDA, S.; BERRUTI, F. Municipal solid waste management and landfilling technologies: a review. Environmental Chemistry Letters, v. 19, p. 1433-1456 2020. DOI: https://doiorg/10.1007/s10311-020-01100-y

PETERSEN, P. F.; SILVEIRA, L. M. Agroecology, public policies and labor-driven intensification: Alternative development trajectories in the Brazilian semi-arid region. Sustainability, v. 9, n. 4, p. e535, 2017. DOI: https://doi.org/10.3390/su9040535

PIGIRS - Plano Intermunicipal de Gestão Integrada de Resíduos Sólidos. Região Sertão. Maceió: SEMARH, 2016. 58 p.

R DEVELOPMENT CORE TEAM. R Foundation For Statistical Computing. R: A Language and Environment for Statistical Computing. Viena, Áustria. 2019. ISBN: 3-900051-07-0. Disponível em: http://www.R-project.org. Acesso em 10 de maio de 2021.

RAMACHANDRA, T. V.; BHARATH, H. A.; KULKARNI, G.; HAN, S. S. Municipal solid waste: Generation, composition and GHG emissions in Bangalore, India. Renewable and Sustainable Energy Reviews, v. 82, p. 1122-1136, 2018. DOI: https://doi.org/10.1016/i.rser.2017.09.085

SANTOS, P. C.; ROCHA, A. C. M. A.; LIMA, E. D. S.; SANTOS, J. P. O.; GONZAGA, K. S.; CARTAXO, P. H. A.; SANTOS, A. S.; BULHÕES, L. E. L.; MORAIS, W. 
R. S. Generation of urban solid waste: application of a sustainability indicator in a municipality of the Alagoas semi-arid. Research, Society and Development, v. 9, n. 8, p. e244985661, 2020. DOI: https://doi.org/10.33448/rsd-v9i8.5661

WEI, Y.; LI, J.; SHI, D.; LIU, G.; ZHAO, Y.; SHIMAOKA, T. Environmental challenges impeding the composting of biodegradable municipal solid waste: A critical review. Resources, Conservation and Recycling, v. 122 , p. $51-65,2017$. DOI: https://doiorg.ez15.periodicos.capes.gov.br/10.1016/j.resco nrec.2017.01.024 